\title{
Treatment against Mutation of PIK3CA Gene Involved in Lung Cancer by Structure Base Pharmacophore Modeling, Virtual Screening and Molecular Docking
}

\author{
Zunera Turk*, Muhammad Hamza, Anum Munir, Sajid Khan, Muhammad Rizwan, Azhar Mehmood \\ Govt Post Graduate College Mandian, Pakistan
}

Received August 19, 2019; Revised October 14, 2019; Accepted October 20, 2019

Copyright $\odot 2019$ by authors, all rights reserved. Authors agree that this article remains permanently open access under the terms of the Creative Commons Attribution License 4.0 International License

\begin{abstract}
Lung Cancer is a type of serious cancer that causes 1.37 million deaths every year all over the world and accounts for almost $18 \%$ of all cancer death. PI3Ks establish a lipid kinase family that is important to integrate disparate dimensions of cell functions including cell survival, vesicular trafficking, proliferation, and cell migration. This is an essential pathway in the oncogenesis and advancement of lung cancer. In preclinical studies, PIK3 inhibitors deliver exploratory antitumor activity. The study was established to realize perception and molecular mechanisms that are crucial for potent inhibitors of PIK3CA. In this research work, mutated proteins of PIK3CA were selected, models of pharmacophore were designed and hit compounds were obtained against reference feature pharmacophore by virtual screening. These hit compounds were then docked with the mutated proteins of PIK3CA. Three important features were shown by Pharmacophores, Hydrogen bond donor (HBD), Hydrogen bond acceptors (HBA) and aromatic rings (AR). Through virtual screening, 8 hit compounds were obtained before docking Lipinski rule of five was applied and the compounds that achieved all properties were docked with mutated proteins of PIK3CA. 3 compounds fulfilled all properties and demonstrated the stability of ligands. It is suggested that these compounds can be used for curing PIK3CA involved in lung cancer and on the basis of shared feature novel compounds can be designed against a mutation in PIK3CA involved in lung cancer.
\end{abstract}

Keywords Pharmacophores, HBA, HBD, Virtual Screening, Ideal Docking

\section{Introduction}

Lung Cancer is a serious type of cancer that causes 1.37 million death every year worldwide, which accounts for almost $18 \%$ of all cancer death. [1] The most common cause of lung cancer is smoking. About $90 \%$ of lung cancer occurs as an outcome of smoking tobacco. Lung Cancer is generally classified into two types. Small Cell Lung Cancer (SCLC) accounts for 10-15\% and Non-Small Cell Lung Cancer (NSCLC) which accounts for approximately $80 \%$. [2] NSCLC is a leading factor causing death worldwide. [3] The phosphoinositide 3-kinases (PI3Ks) establish a lipid kinase family that is important to integrate disparate dimensions of cell functions including cell survival, vesicular trafficking, proliferation, and cell migration. This is an important pathway in the oncogenesis and advancement of lung cancer. In preclinical studies, PIK3 inhibitors deliver exploratory antitumor activity. [4] NSCLC has four different advance stages. Cancer at stage I is located in the lungs and has not transmitted to any lymph nodes. At Stage II cancer has spread to nearby lymph nodes while at Stage III, cancer has spread to the center of chest, 2 subtypes. i-e: Stage IIIA and Stage IIIB and stage IV is the leading stage in which cancer has spread to both lungs. PI3K (phosphatidylinositol 3 kinase) plays an essential role in the proliferation and metabolism of cell that typically affects both cancer and metabolic disorder. [5,6,7]

Cancer at the initial level of prognosis shows an important appearance in the identification mechanism and for an efficient planning procedure approach. [8] Generally in most advanced stages of NSCLC, when diagnosed initially, surgical abscission applied is not suitable and useful. [9] The patient may have an opportunity of continuous survival chemotherapy as well for advanced leading NSCLC is generally recognized as extremely toxic and virulent. [10]

Pharmacophore modeling is a very prospering and successful subfield of computer-aided drug design and these approaches have become successful after the development of the past century. Ligand-based and 
Structure base procedures are established to enhance pharmacophore modeling. The concept of the pharmacophore has been widely applied to virtual screening, de novo design and the rational style of novel medicine. [11] The first step to realizing the interaction between receptor and ligand molecule is pharmacophore. During the late 1800s, Paul Ehrlich developed the concept of pharmacophore. [12] Pharmacophore may be used to align the molecules based on the three-dimensional arrangement of chemical structures.

\section{Materials and Methods}

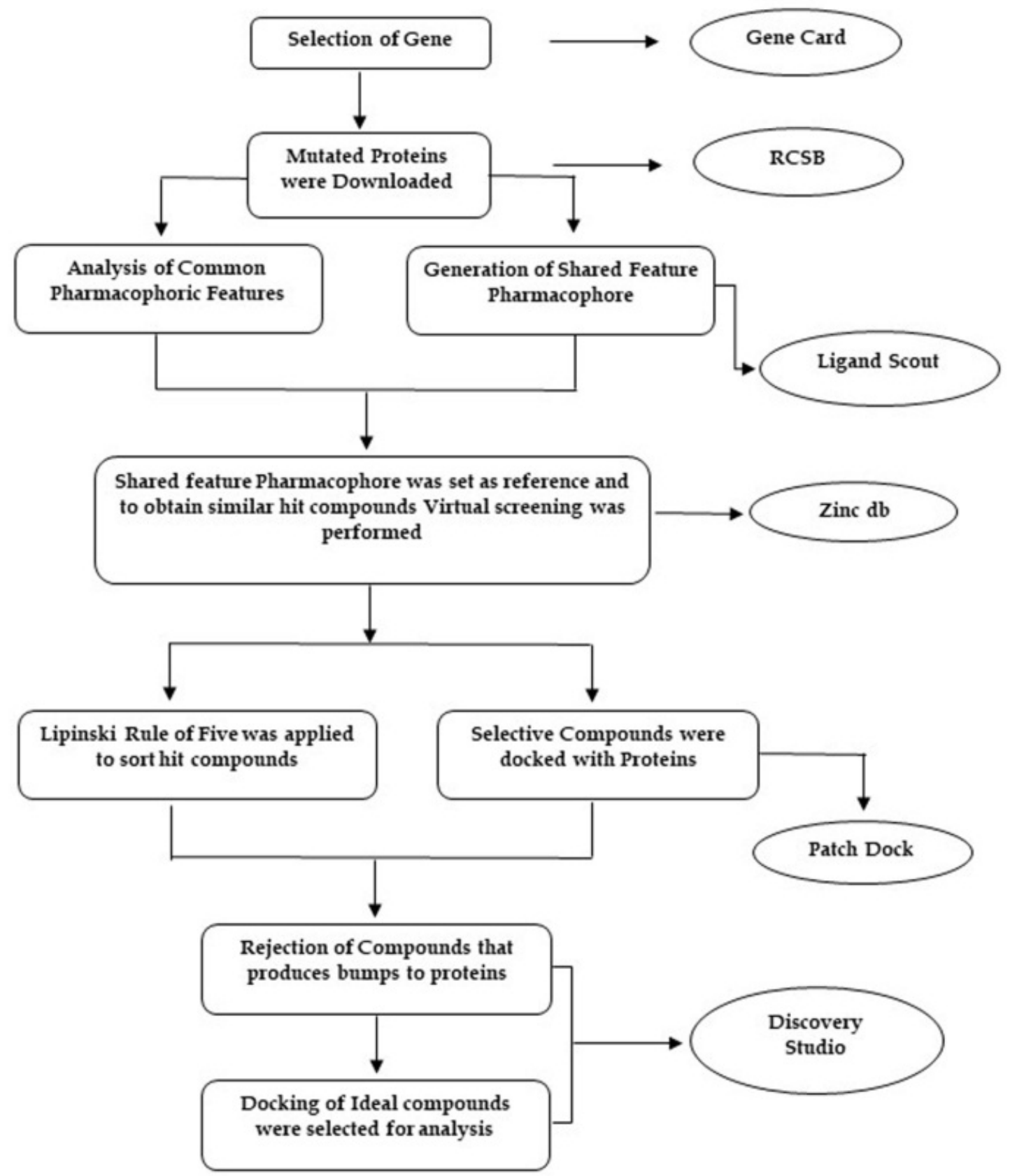

Figure 1. Method for Designing Pharmacophore Model against PIK3CA mutation in Lung Cancer 


\subsection{Selection of Proteins}

Gene PIK3CA was selected from Gene cards data base (https://www.genecards.org/) Mutated Proteins of the gene were downloaded from Protein data bank (Pdb). Five mutated proteins were selected that were homo sapiens with the experimental method of X-Ray crystallography with an X-Ray resolution of 2.0-3.0. The proteins named as 5UK8, 5UKJ, 5UL1, 5ITD, 4ZOP.

\subsection{Protein Preparation}

Six mutated proteins of gene PIK3CA involved in lung cancer were downloaded by using Pdb (www.rcsb.org). $5 \mathrm{UK} 8$ is the co-structure of (R)-4-(6-(1-(cyclopropyl sulfonyl) cyclopropyl)-2-(1H-indol-4-yl) pyrimidine-4-yl)-3-methyl morpholine and a rationally designed PI3K-alpha mutant that mimics ATR. It contains 9 mutations. $5 \mathrm{UKJ}$ is the co-structure of $\mathrm{N}$, N-dimethyl-4-[(6R)-6-methyl-5-(1H-pyrrolo [2,3- b] pyridin-4-yl)-4,5,6,7-tetra hydro pyrazolo [1,5a]pyrazin-3-yl]benzene sulfonamide and a rationally designed PI3K-alpha mutant that mimics ATR, also contains 9 mutations. 5UL1. The co-structure of 3-amino-6-(4-((1-(dimethyl amino) propan-2-yl) sulfonyl) phenyl)-N-phenylpyrazine-2-carboxamide and a rationally designed PI3K-alpha mutant that mimics ATR along with 9 mutations. 5ITD is a crystal structure of PI3K alpha with PI3K delta inhibitor along with 2 mutations. All of these are transferase signaling protein inhibitors. 4ZOP Co-crystal Structure of Lipid Kinase PI3K alpha with a selective phosphatidylinositol-3 kinase alpha inhibitor along with 2 mutations and is Transferase/Transferase inhibitor.

\subsection{Generation of Pharmacophore}

By importing all these mutated proteins into Ligand Scout, common pharmacophoric features of every ligand were checked along with their interactions and shared feature pharmacophore was designed. [12] Shared Feature Pharmacophore contains some essential features i-e: Hydrogen Bond Donors, Hydrogen Bond Acceptors,
Aromatic Rings, and Hydrophobic structures.

\subsection{Virtual Screening}

Shared Feature Pharmacophore model was set as a reference by exporting into alignment tab of Ligand Scout. By importing Zinc library into ligand scout, virtual screening was performed for obtaining similar hit compounds with shared feature pharmacophore. [13]

\subsection{Acquiring Lipinski Rule of Five for validating Hit Compounds}

Lipinski Rule of five was checked for the obtained compoudns after virtual screening. Following are the Rule of Five for any compound for validation: Hydrogen Bond Donor should be less than 10, Hydrogen Bond Acceptor should be less than 5 , $\log \mathrm{P}$ value ranges in between $0-5$ and Molecular Weight should never exceed than 500. The toxicity class should be 4 or above. [14]

\subsection{Docking}

Docking was performed after screening the compounds form Lipinski Rule of five, Compounds that fulfill the Lipinski Rule of Five were selected and docked with the mutated Proteins by using the Patch Dock server (bioinfo3d.cs.tau.ac.il/PatchDock). Docking results were analyzed by using Discovery studio. [15]

\subsection{Results}

A total of 5 proteins were selected named as 5UK8, 5UKJ, 5UL1, 5ITD, 4ZOP. The 3D structures of selected proteins along with ligands are shown in figure 2. 


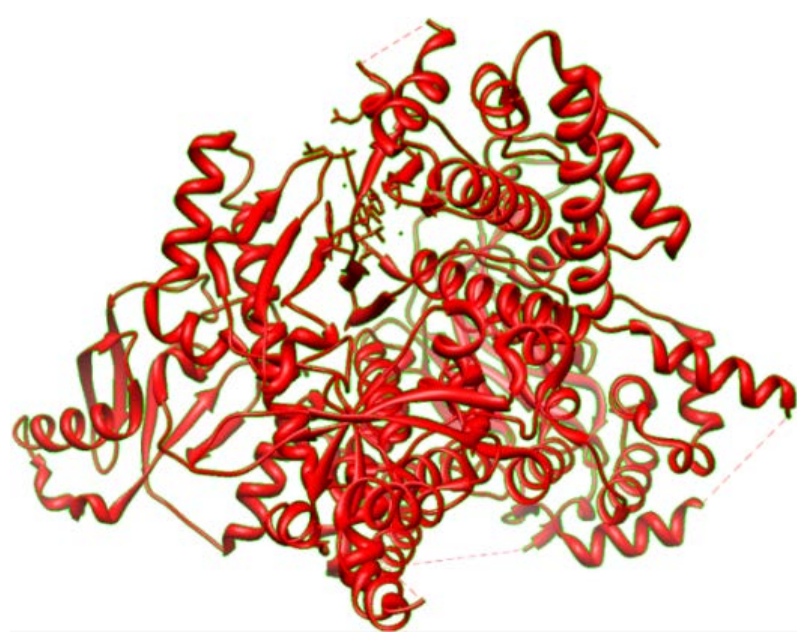

a) $5 \mathrm{UKJ}$

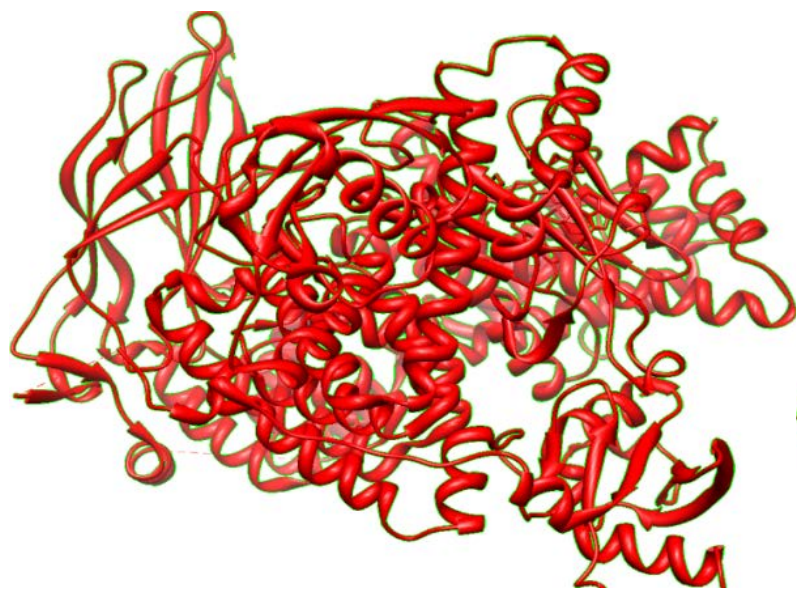

c) 5 UK8

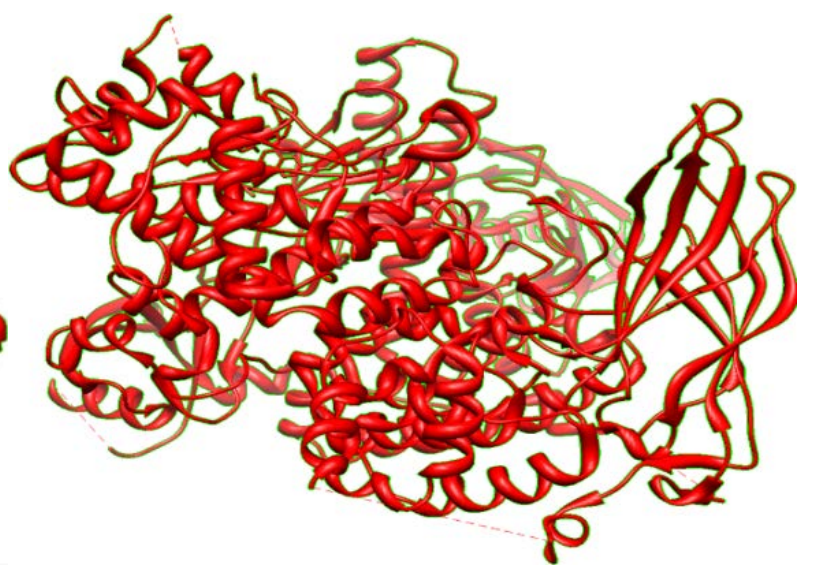

b) 5 ITD

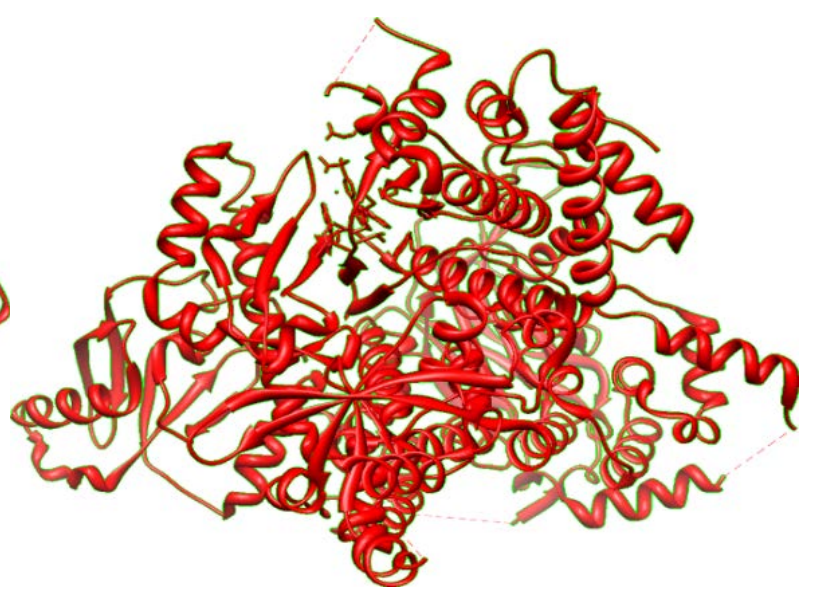

d) 5UL1

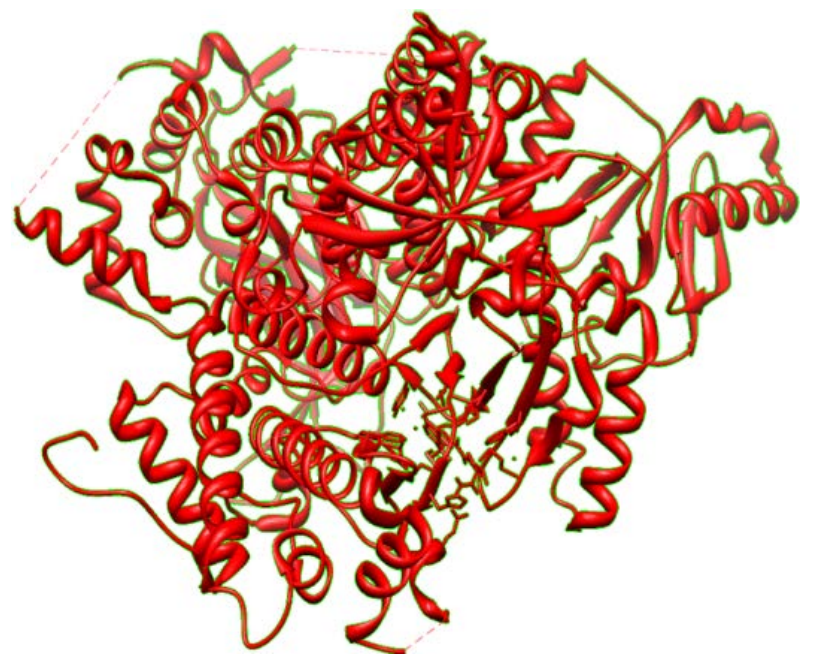

e) $4 \mathrm{ZOP}$

Figure 2. 3-D Structure of Proteins along with Ligands 
5UKJ consist of 1266 amino-acids along with 2 unique chains A and B. 5ITD consist of 1232 amino-acids with 2 chains, 5UK8 consist of 1252 amino-acids along with two chains A and B. 5UL1 consist of 1257 amino-acids along with two specific chains. Similarly, 4ZOP also contains two chains A, B, and 1240 amino-acids residues.

The Pharmacophore designs of each of the protein is shown in figure 3-7.

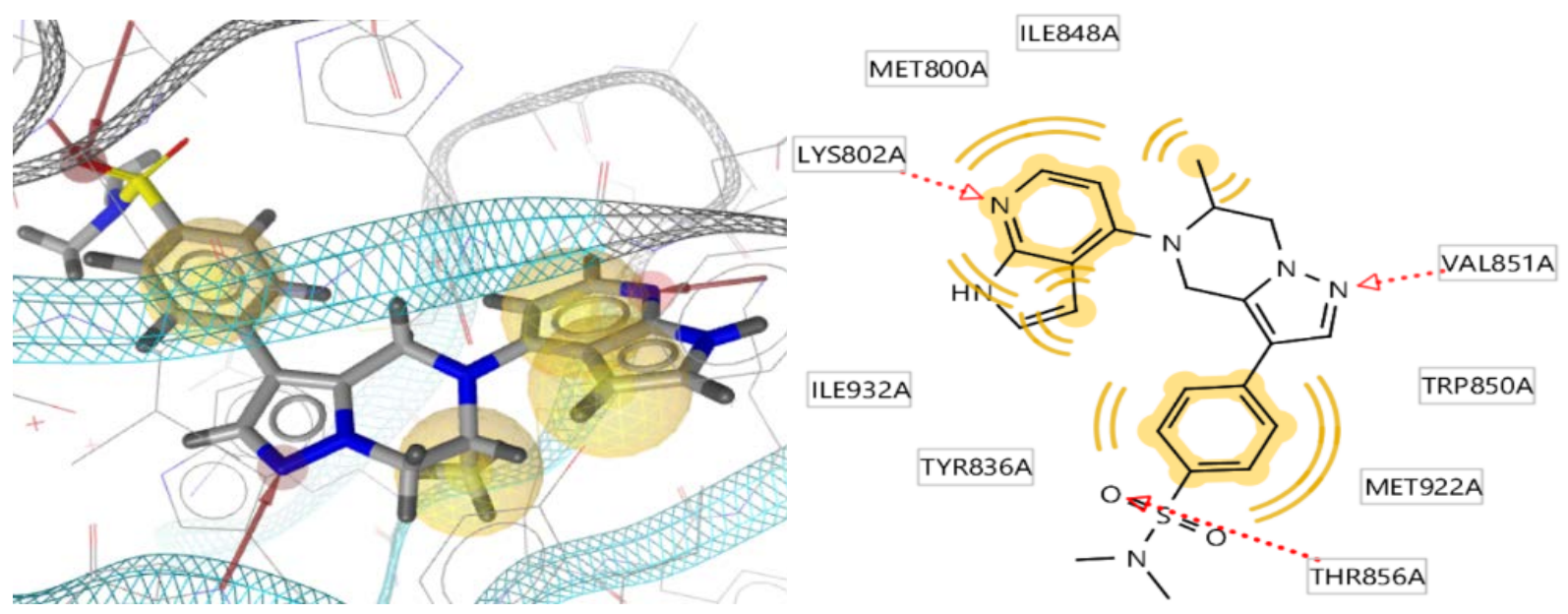

Figure 3. Pharmacophore and Interactions of 5UKJ
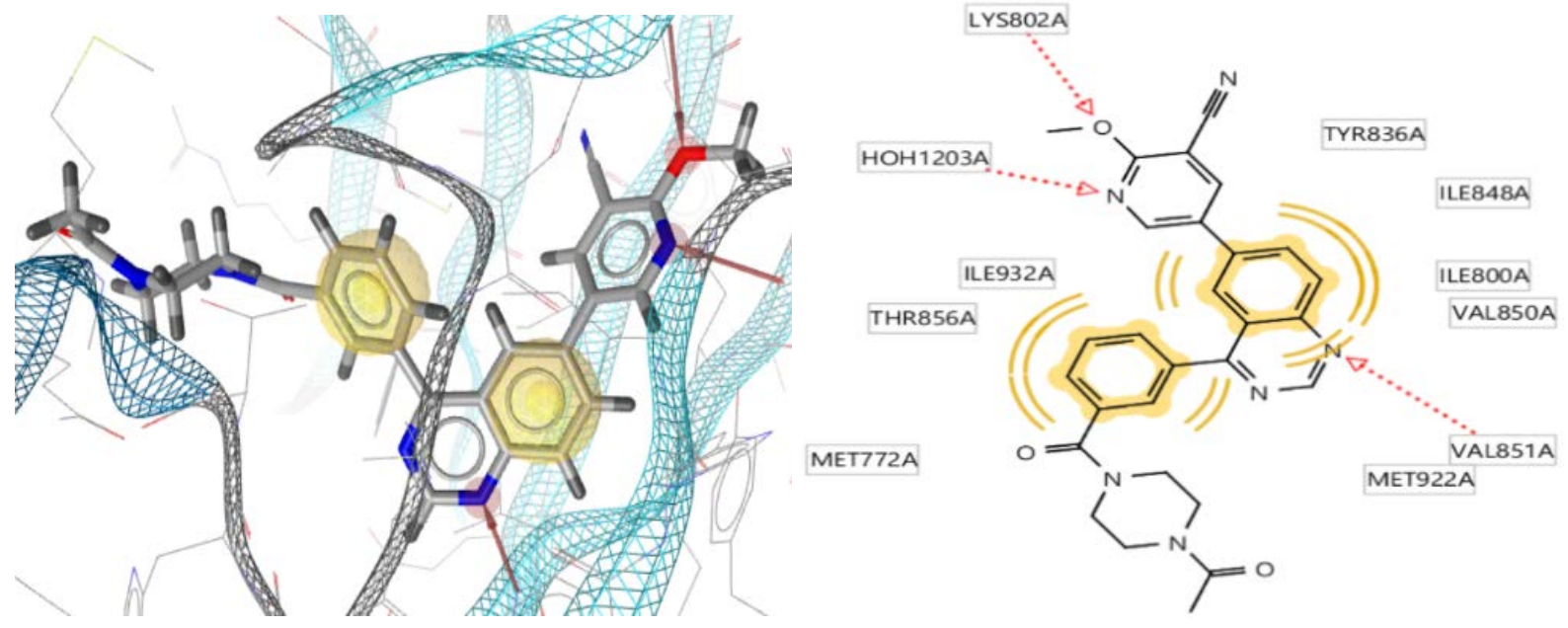

Figure 4. Pharmacophores and Interactions of 5ITD 

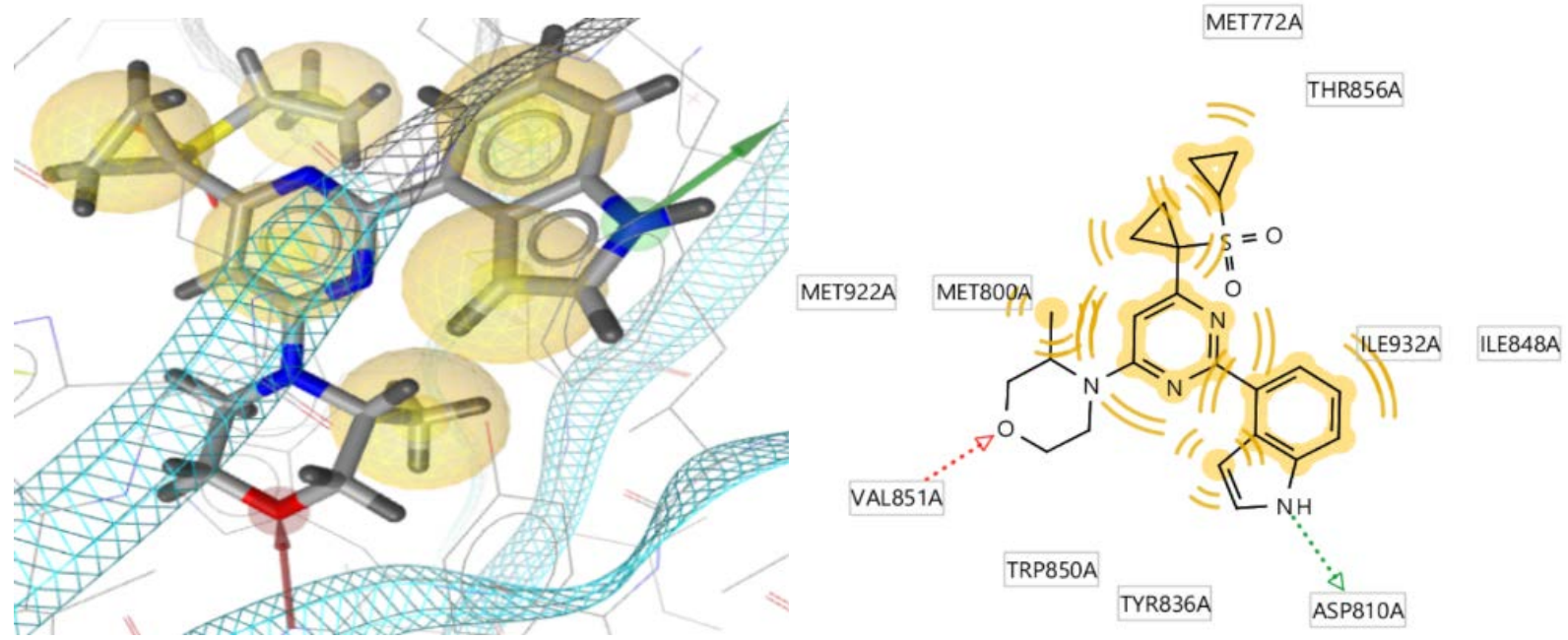

Figure 5. Pharmacophore and Interactions of 5UK8

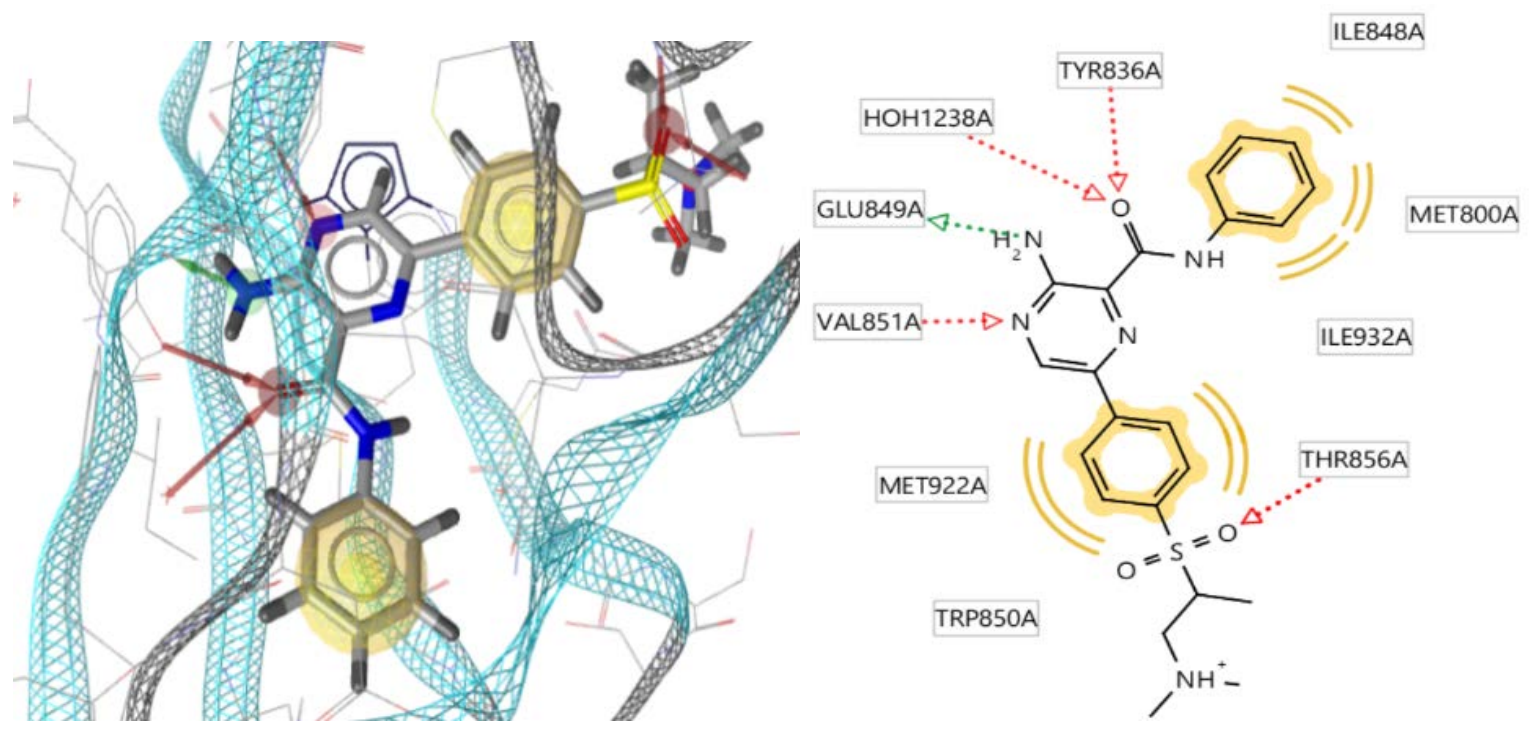

Figure 6. Pharmacophore and Interactions of 5UL1
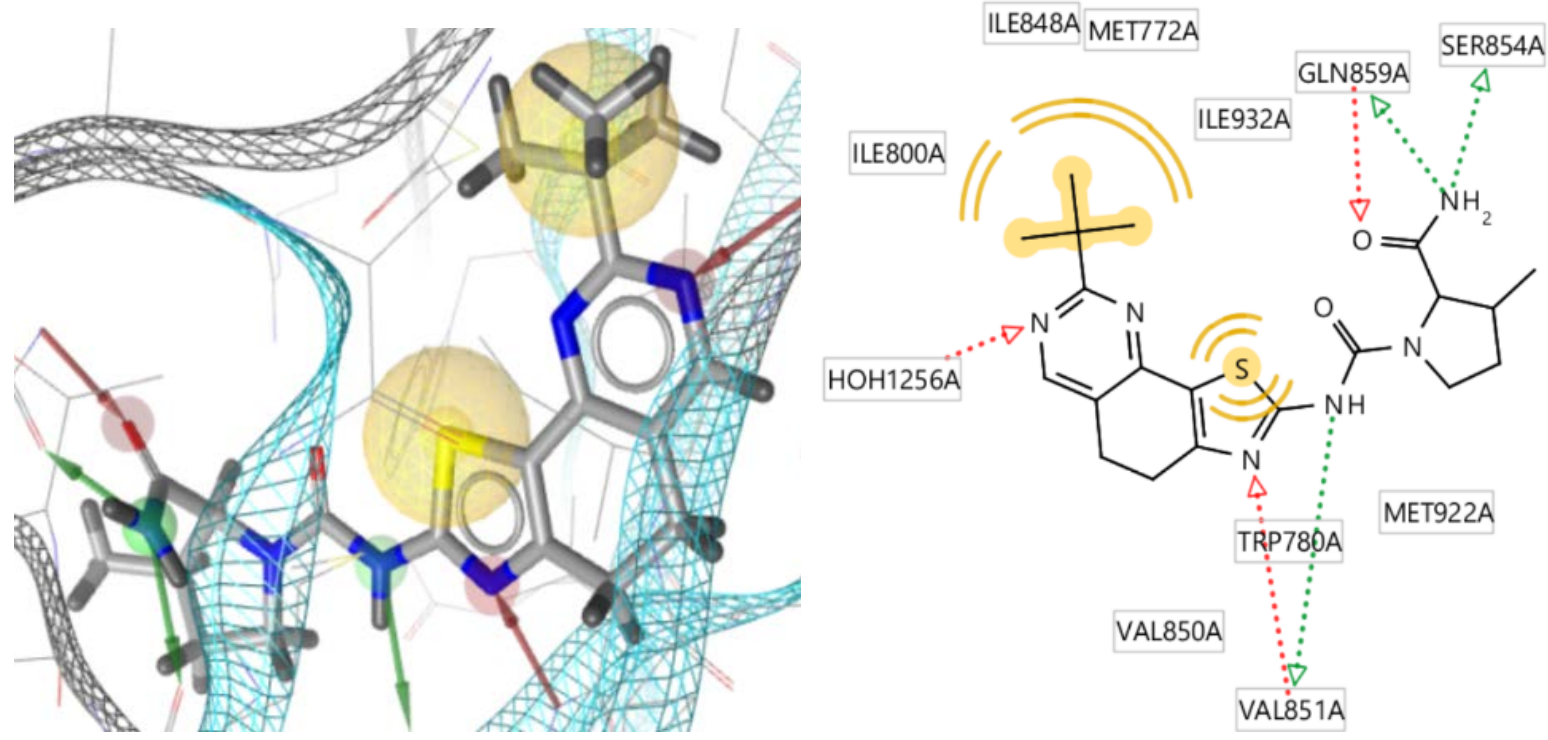

Figure 7. Pharmacophore and Interactions of $4 \mathrm{ZOP}$ 
In figure 3-7, Pharmacophores designed by ligand scout against the protein data set of PIK3CA in lung cancer show three significant features that are HBA, HBD, and AR. Red arrows represent HBA, the green arrow represents HBD and AR was shown by yellow spheres.

The shared feature pharmacophore model is shown in figure 8

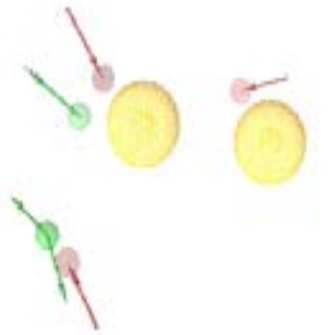

f)

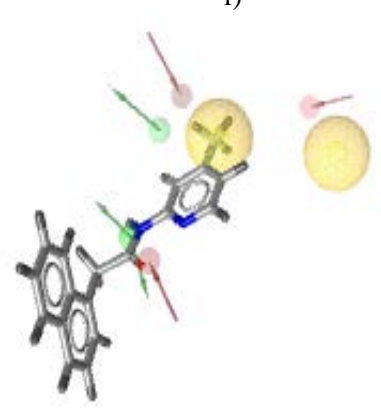

h)

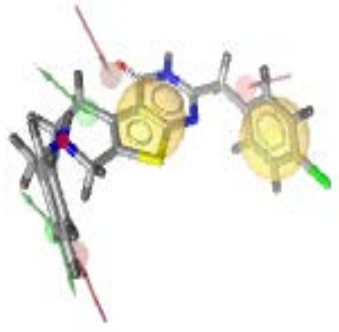

g)

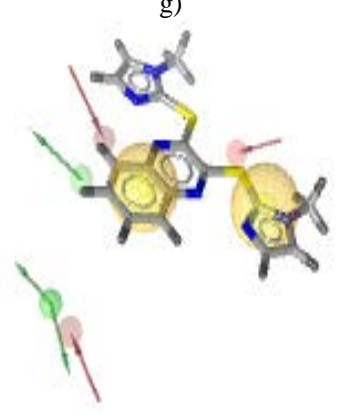

i)
Figure 8. f) shared feature pharmacophore, g) ZINC08442270, h) ZINC 09365179, i) ZINC05286115
In Figure 8 Virtual Screenings of shared feature pharmacophore model, 8 hit compounds were obtained that were similar to shared feature pharmacophore. Lipinski Rule of Five was applied to each hit compound. Only three compounds fulfill all aspects of the rules and demonstrate to the toxicity class 4. ZINC08442270, ZINC 09365179, ZINC05286115. These compounds were downloaded and converted into PDB format by using Discovery Studio and used as a ligand for docking with mutated proteins in Patch Dock.

After virtual screening, eight-hit compounds were obtained, which were further sorted for the Lipinski Rule of Five. Three compounds fulfill all rules that were: Hydrogen Bond Donor should be less than 10, Hydrogen Bond Acceptor should be less than $5, \log \mathrm{P}$ value ranges in between 0-5 and Molecular Weight should never exceed than 500. The toxicity class should be 4 or above. Lipinski rule of five for each compound after virtual screening is shown in table 1.

Compounds that satisfy Lipinski Rule of Five were selected and docked with the mutated Proteins by using the Patch Dock server (bioinfo3d.cs.tau.ac.il/PatchDock). Five proteins were selected as receptor proteins while 3 selected as ligand upon the basis of pharmacophore fit score. Patch Dock is used for molecular docking, docking results were analyzed by using Discovery studio. 2-D interactions were also analyzed there and the compounds that produce bumps to proteins were discarded and the compounds that show ideal docking were selected. Docking results are shown in figure 9 and 10.

Table 1. Compounds that fulfill Lipinski Rule of and taken as Ligand

\begin{tabular}{|l|l|l|l|l|}
\hline Compounds & Molecular Weight & Log P & HBD & HBA \\
\hline & & & & \\
\hline
\end{tabular}



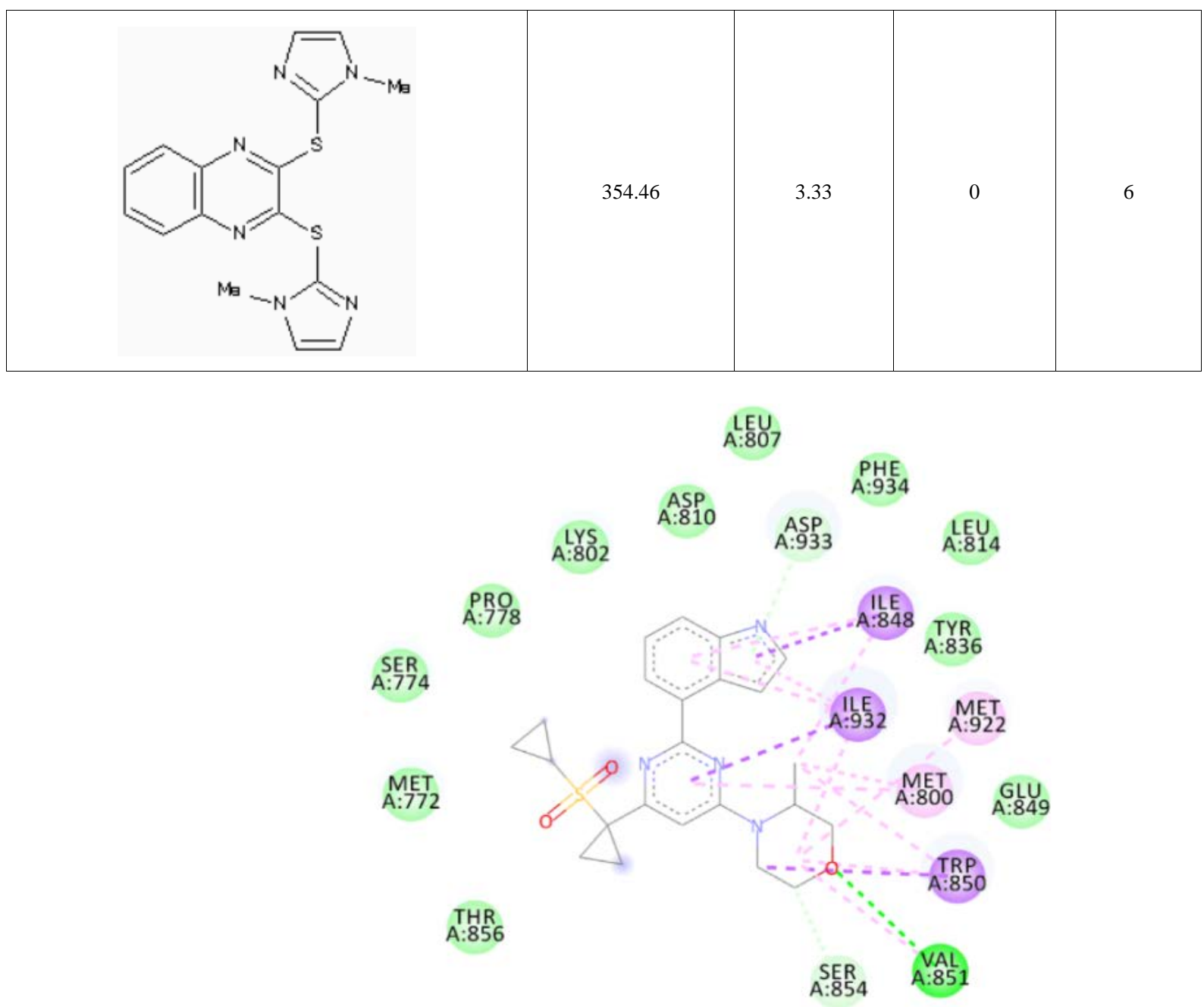

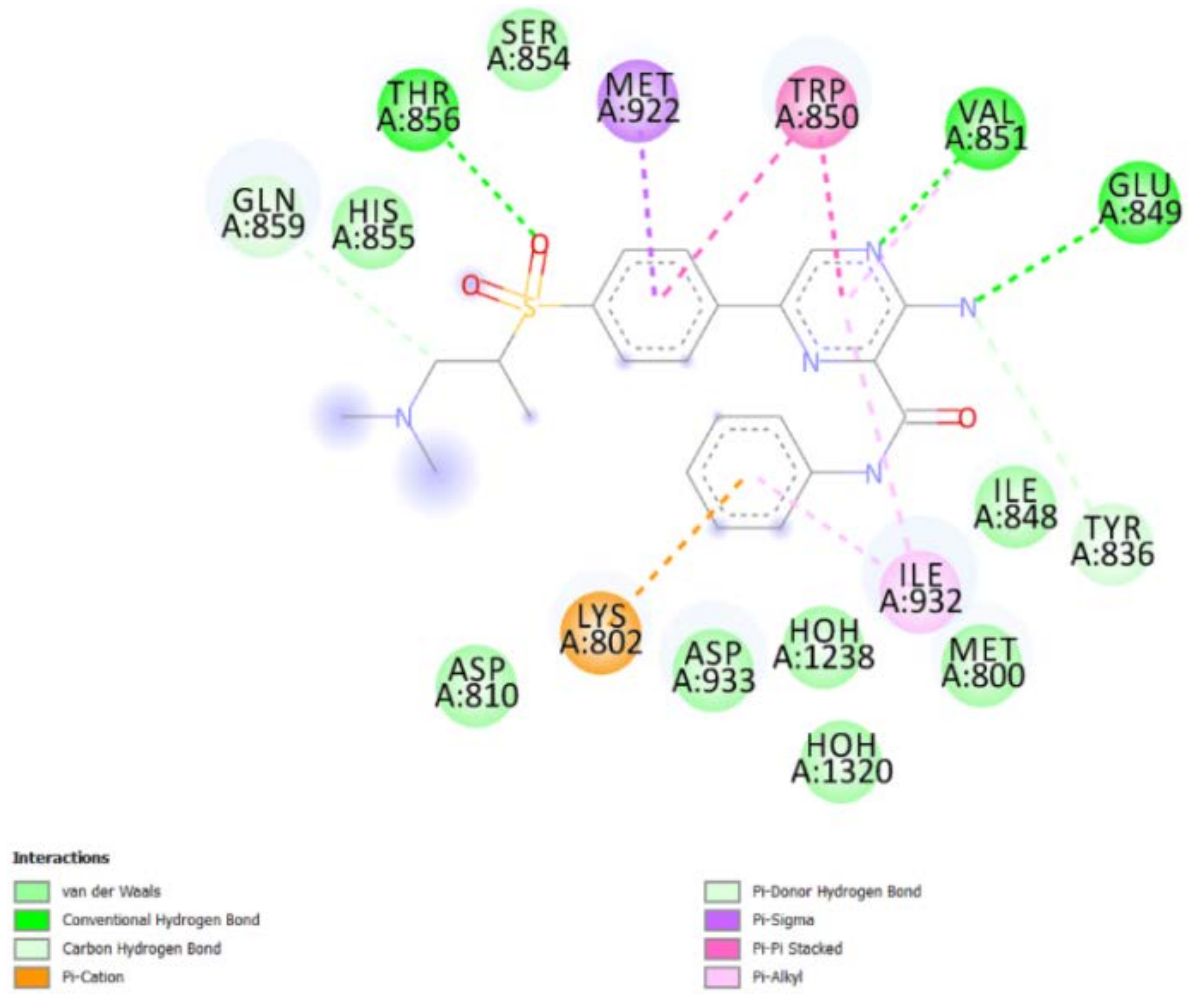

b)
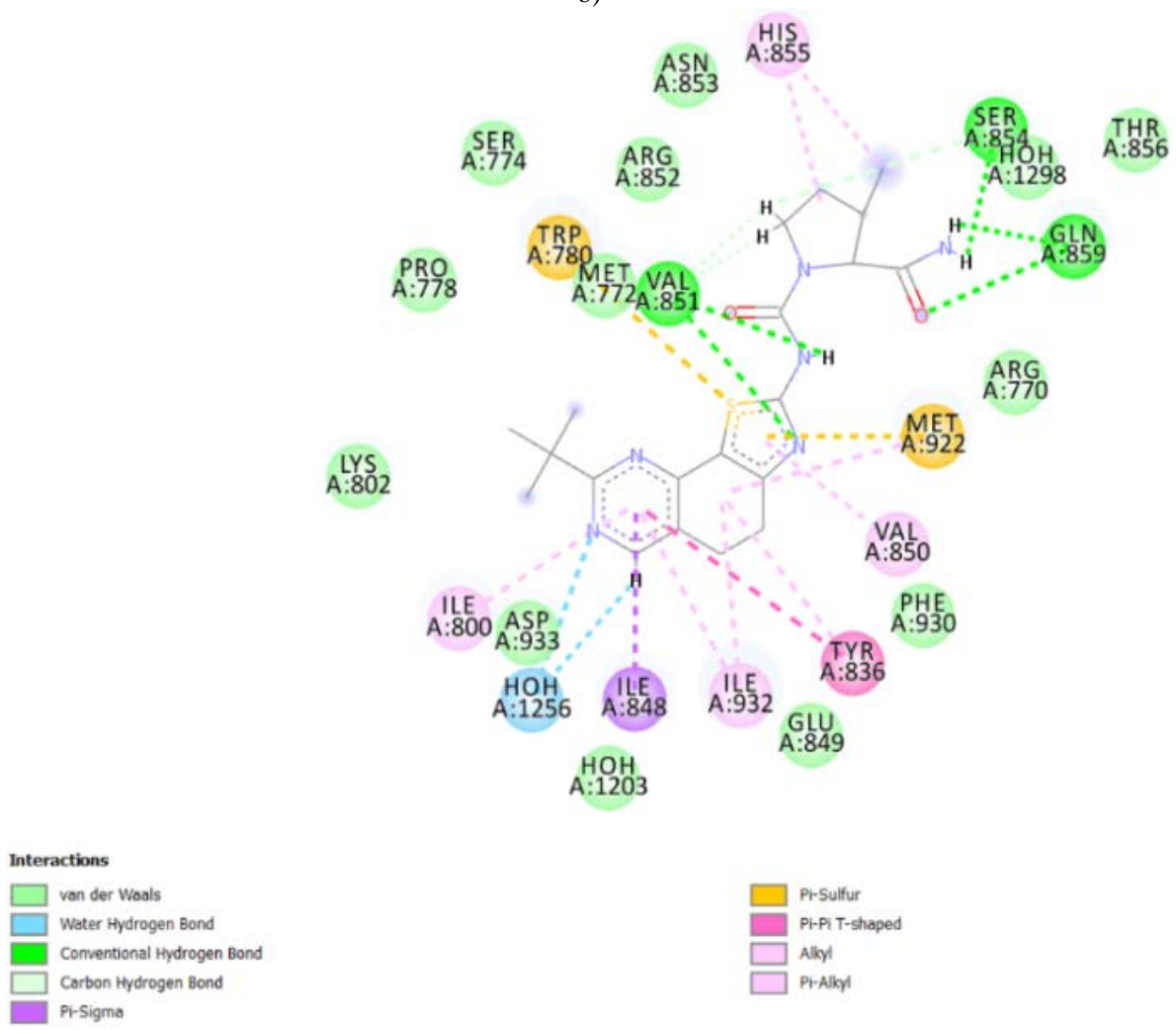

c) 

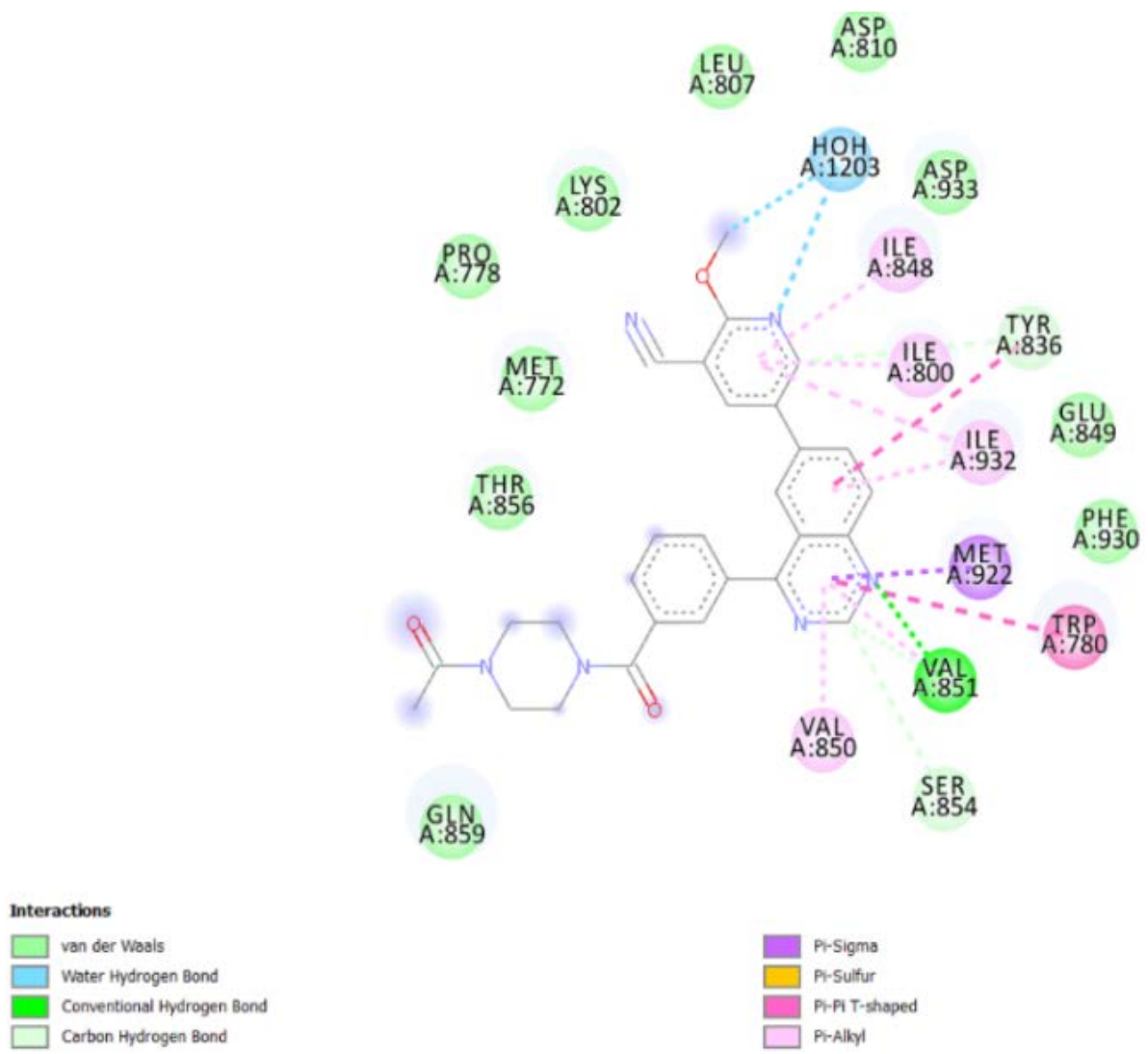

d)

Figure 9. Resulted Compounds that demonstrate ideal Docking Interactions

LEU

A:473

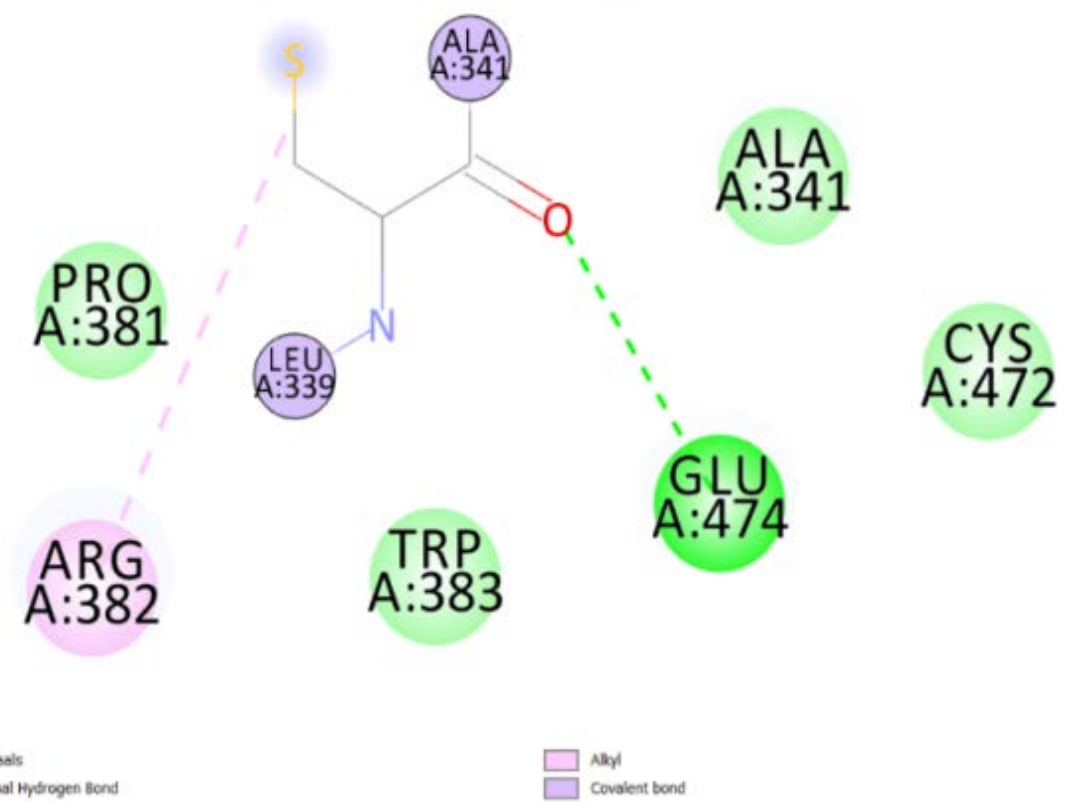

e) 


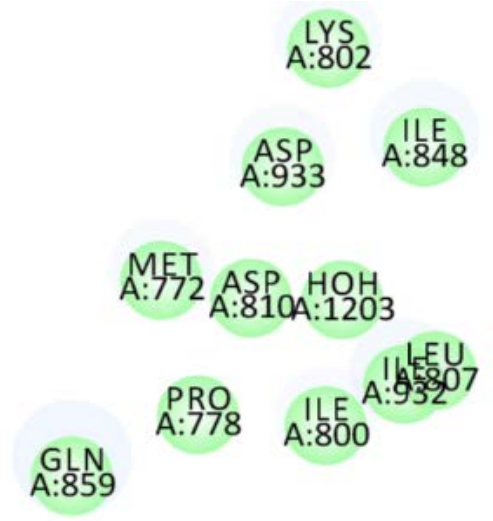

A: 851
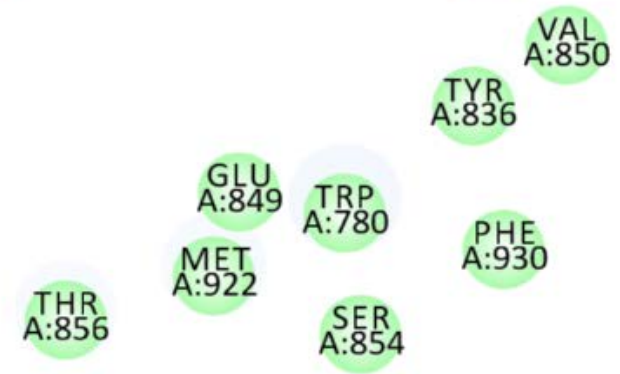

Interactions

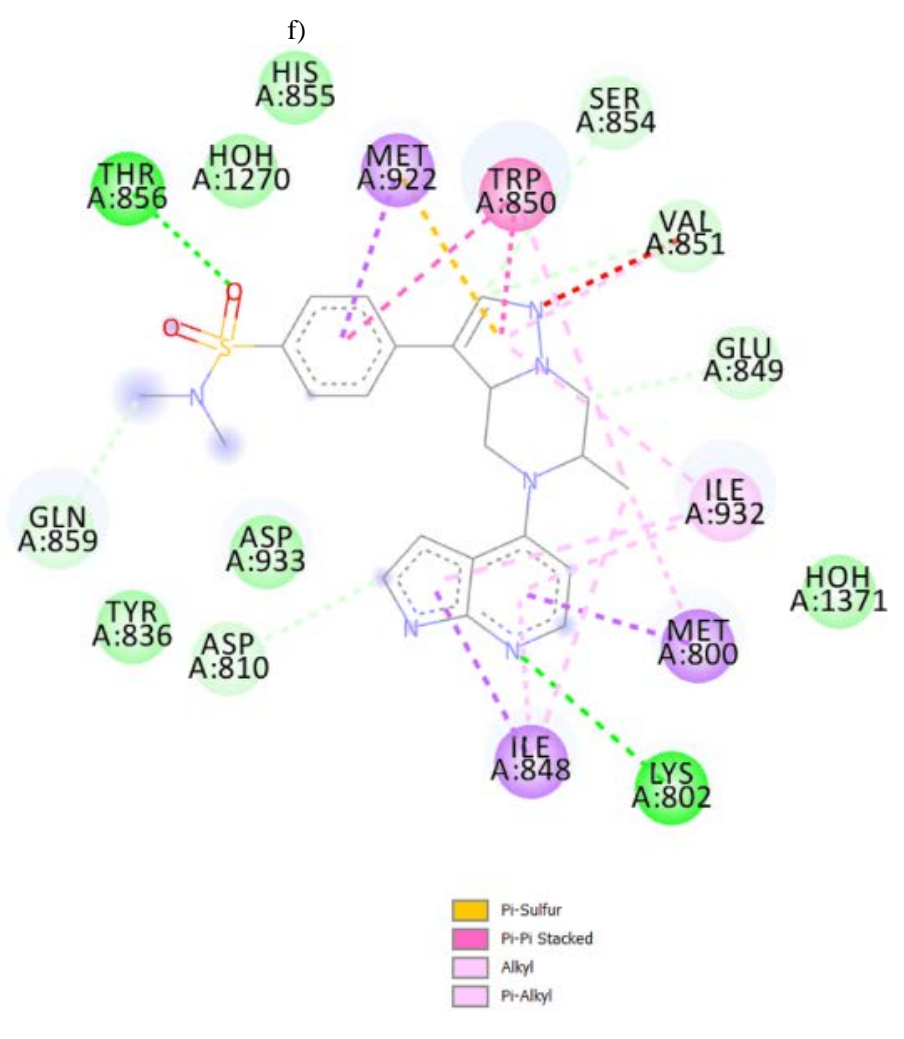

g) 


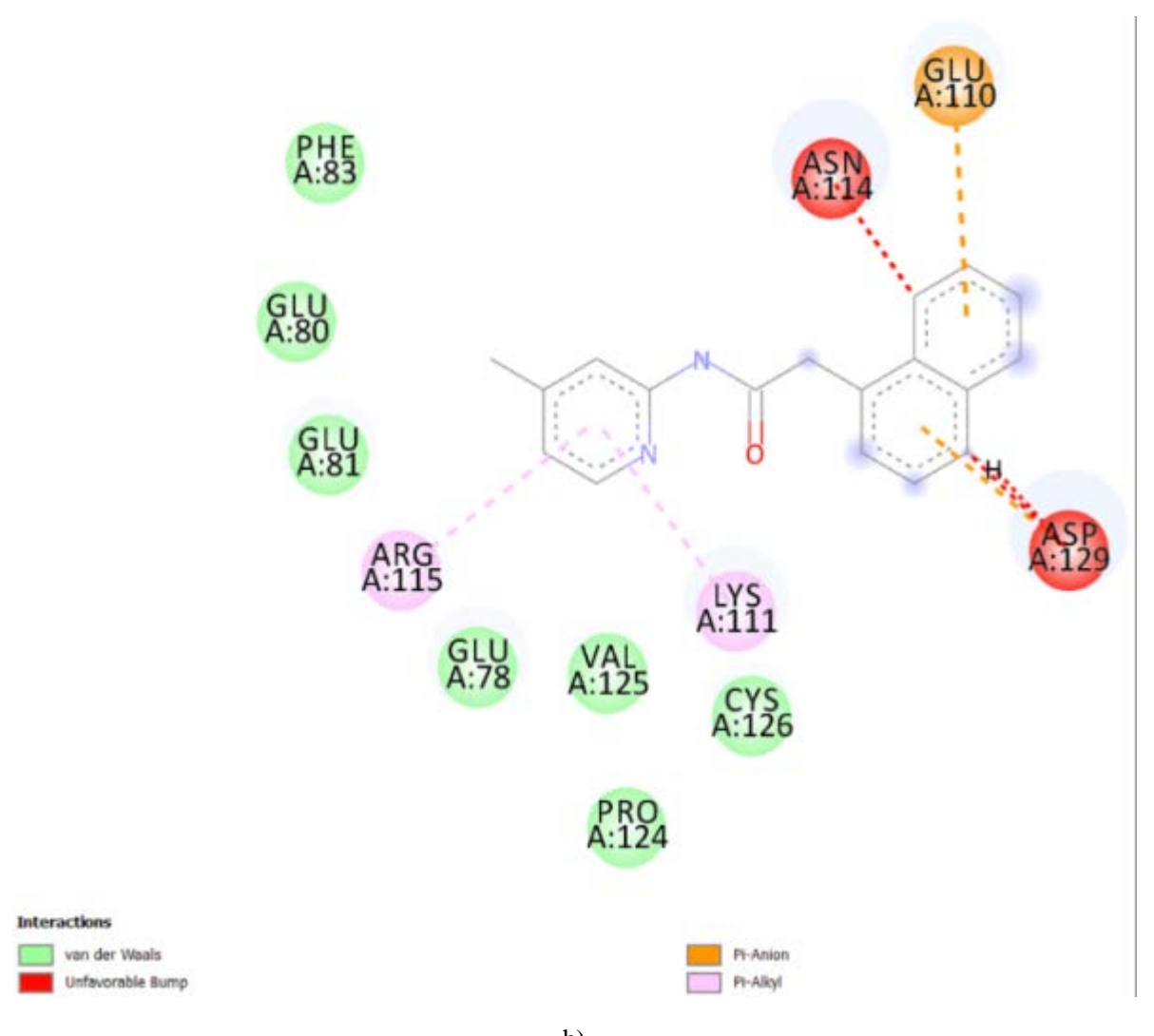

h)

Figure 10. Compounds that show bumps and few interactions were rejected because they are not Ideal

Docking Results were analyzed in Discovery Studio and compounds showing ideal docking without creating any bumps were selected. 2-D interactions were also visualized.

\section{Discussions}

The pharmacophore modeling is an affective approach for the development of new leading and emerging compounds. Arrangements of chemical-based on the 3-D structure of a molecule may be aligned by using pharmacophore modeling. It is the key step to understand the interaction between a receptor and a ligand. Pharmacophores are usually used to derive an essential relation between structure and set of active molecules. The most common approaches of pharmacophore modeling are structure-based and ligand base. A set of active compounds should be deposited and shared chemical features necessary for the bio-activity of molecules should be extracted while in structure-based pharmacophore modeling interactions between receptor and ligand molecule should be directly examined to a promising extent. [16,17]

In this structure base pharmacophore modeling research work, the combination of pharmacophore by virtual screening and molecular docking beneficently influences all possible inhibitors for different studies in disease experimentally. [18] we analyze different chemical features of compounds and are best to interpret the structure base models as well as the interaction between ligand molecule and target receptor of protein. Virtual Screening is a high throughput screening technique, and this may be done by screening ligand libraries in approaches to drug discovery. [19] Compounds obtained by virtual screening were sorted for Lipinski rule of five to gather top compound against lung cancer and finally, 3 compounds were selected for docking. Molecular Docking is used to analyzing further hit compounds as an inhibitor against PIK3CA. Among the docked results compounds that produce bumps were rejected.

Several Inhibitors such as Abemaciclib, Pemetrexed, Gemcitabine, Ramucirumab, and Pembrolizumab are being already used to treat PI3K mutations but number of side effects are reported in literature due to their use as a treatment measure, whereas the compound reported in this study is least toxic as it fulfills all the properties of drug like compounds and is non-toxic in nature. The use of single-agent PI3K inhibitors achieved modest responses and is unlikely to be a curative therapy for diverse cancers. The efficacy of already used PI3K inhibitors is limited for their narrow therapeutic window and frequent 
treatment-related toxicities [20]. The pharmacophore inhibitors of PIK3CA, will significantly bind to its active site. As, multiple strategies were applied to accomplish the inhibition of basic structural distinctive for PIK3CA.

Virtual Screening and Structure Base Pharmacophores aid to trigger the biological activity of compounds along with changes in chemical features and offers an effective location for designing novel inhibitor compounds. The specific hypothesis achieved due to the interaction between the receptor-ligand complex in structure base model [21]s. Drugs available in the market are toxic and some of them also have side-effects while drugs obtain to inhibit PIK3CA(NSCLC) lung cancer by using structure-based pharmacophore modeling are less toxic and have very rare side effects as compared to market drugs. The drug will be directly bound to the target mutated protein and will be cured easily and efficiently in less time.

\section{Conclusions}

In-silico method is used in this research work to find the inhibitor of PIK3CA, which will significantly bind to its active site. Multiple strategies were applied to accomplish the inhibition of basic structural distinctive for PIK3CA. Pharmacophores designed by applying the configuration to the pharmacophore model, virtual screening and molecular docking contain three primary features; HBD, HBA, and AR. In the future, these pharmacophores can be tested in-vitro against PIK3CA to test its advantages. To increase the efficacy of these pharmacophores as inhibitors compounds, further experimental validation may be conducted.

\section{Acknowledgements}

None of the authors have any challenging conflict of interest. The authors are grateful to the department of Bioinformatics Govt. Postgraduate College Mandian, Abbottabad and Bioinformatics International Research Club GPGCM, for providing a platform to conduct the research.

\section{REFERENCES}

[1] World Health Organization. Cancer. Fact Sheet Number 297. www. who.int/mediacentre/factsheets/fs297/en/

[2] Molina, J. R., Yang, P., Cassivi, S. D., Schild, S. E., \& Adjei, A. A. Non-small cell lung cancer: epidemiology, risk factors, treatment, and survivorship. Mayo Clinic Proceedings Vol. 83, No. 5, pp. 584-594, 2008.

[3] Ginsberg RJ, Vokes EE, Raben A. In: Cancer: Principles \& Practice. 8. De Vita VT, Hellman S, Rosenberg SA, editor.
Lippincott Williams \& Wilkins; 1997. Cancer of the lung, section 2: non-small cell lung cancer; p. 858.

[4] Wu, S. G., Chang, Y. L., Yu, C. J., Yang, P. C., \& Shih, J. Y. The Role of PIK3CA mutations among lung adenocarcinoma patients with primary and acquired resistance to EGFR tyrosine kinase inhibition. Scientific reports, Vol. 6, 35249, 2016.

[5] Scheffler, M., Bos, M., Gardizi, M., König, K., Michels, S., Fassunke, J., ... \& Albus, K. PIK3CA mutations in non-small cell lung cancer (NSCLC): genetic heterogeneity, prognostic impact and incidence of prior malignancies. Oncotarget, Vol. 6, No. 2, 1315, 2015

[6] Yuan, T. L., \& Cantley, L. C. PI3K pathway alterations in cancer: variations on a theme. Oncogene, 27(41), 5497, 2008

[7] Courtney, K. D., Corcoran, R. B., \& Engelman, J. A. The PI3K pathway as drug target in human cancer. Journal of clinical oncology, Vol. 28, No. 6, 1075, 2010

[8] Ahmed, K., Kawsar, A. A., Kawsar, E., Emran, A. A., Jesmin, T., Mukti, R. F., ... \& Ahmed, F. Early detection of lung cancer risk using data mining, Asian Pacific Journal of Cancer Prevention. 2013.

[9] Yang, S. Y. Pharmacophore modeling and applications in drug discovery: challenges and recent advances. Drug discovery today, Vol. 15, No. 11-12, 444-450, 2010

[10] Sun, J. Y., Shi, L., Gao, X. D., \& Xu, S. F. Physical activity and risk of lung cancer: a meta-analysis of prospective cohort studies. Asian Pacific Journal of Cancer Prevention, Vol. 13, No. 7, 3143-3147, 2012

[11] Veselovsky, A. V., Zharkova, M. S., Poroikov, V. V., \& Nicklaus, M. C. Computer-aided design and discovery of protein-protein interaction inhibitors as agents for anti-HIV therapy. SAR and QSAR in Environmental Research, Vol. 25, No. 6, 457-471, 2014

[12] Wolber, G., \& Langer, T. LigandScout: Automated pharmacophore model creation. Bioinformatics, 6020, 2001.

[13] Irwin, J. J., \& Shoichet, B. K. ZINC- a free database of commercially available compounds for virtual screening. Journal of chemical information and modeling, Vol. 45, No. 1, 177-182, 2005.

[14] Pollastri, M. P. Overview on the Rule of Five. Current protocols in pharmacology, Vol. 49, No. 1, 9-12, 2010.

[15] Schneidman-Duhovny, D., Inbar, Y., Nussinov, R., \& Wolfson, H. J. PatchDock and SymmDock: servers for rigid and symmetric docking. Nucleic acids research, Vol. 33, No. suppl_2, W363-W367, 2005.

[16] Yang, S. Y. Pharmacophore modeling and applications in drug discovery: challenges and recent advances. Drug discovery today, Vol. 15, No.11-12, 444-450, 2010.

[17] Kandakatla, N., \& Ramakrishnan, G. Ligand based pharmacophore modeling and virtual screening studies to design novel HDAC2 inhibitors. Advances in bioinformatics, 2014.

[18] Zhang, Y. L., Wang, Y. M., \& Qiao, Y. J. (2013). Structure-based pharmacophore models generation and 
Combinatorial Screening of ICE Inhibitors. In Applied Mechanics and Materials Vol. 347, 1216-1220, 2013.

[19] Group, N. M. A. C. Chemotherapy in addition to supportive care improves survival in advanced non-small-cell lung cancer: a systematic review and meta-analysis of individual patient data from 16 randomized controlled trials. Journal of Clinical Oncology, Vol. 26, No. 28, 4617, 2008.

[20] Yang J, Nie J, Ma X, Wei Y, Peng Y, Wei X. Targeting PI3K in cancer: mechanisms and advances in clinical trials. Molecular cancer. 2019 Dec;18(1):26.

[21] Munir, A., Azam, S., Mehmood, A., Khan, Z., \& Mehmood, A. Structure-Based Pharmacophore Modeling, Virtual Screening and Molecu-lar docking for the Treatment of ESR1 Mutations in Breast Cancer. Drug Des, Vol. 5, No. 137, 2169-0138, 2016. 